\title{
A COMB FILTER DESIGN USING FRACTIONAL-SAMPLE DELAY
}

\author{
Soo-Chang Pei Chien-Cheng Tseng \\ Department of Electrical Engineering, \\ National Taiwan University. \\ Taipei, Taiwan, R. O. C. China \\ Email address: pei@cc.ee.ntu.edu.tw
}

\begin{abstract}
In this paper, a new comb filter design method using fractional sample delay is presented. First, the specification of the comb filter design is transformed into that of fractional delay filter design. Then, conventional FIR and allpass filter design techniques are directly applied to design fractional delay filter with transformed specification. Nex$t$, we develop a constrained fractional delay filter design approach to improve the performance of the direct design method. Finally, several design examples are demonstrated to illustrate the effectiveness of this new design approach.
\end{abstract}

\section{INTRODUCTION}

In many applications of signal processing it is desired to remove harmonic interferences while leaving the broadband signal unchanged. Examples are in the areas of biomedical engineering, communication and control[1]-[5]. A typical one is to cancel power line interference in the recording of electrocardiogram (ECG). Usually, this task can be achieved by the comb filter whose desired frequency response is periodic with small stop-band notches at $0 \mathrm{~Hz}$ to remove base-line wander as well as at $50 \mathrm{~Hz}$ and at its higher harmonics to remove power line disturbance [1]. So far, several methods have been developed to design IIR and FIR comb filters. When the fundamental frequency of harmonic interference is known in advance [1][5], fixed comb filter can be used. However, when fundamental frequency is unknown or time varying, adaptive comb filters are applicable [2]-[4]. In this paper, we will focus on fixed comb filter design problem.

Recently, fractional sample delay has become an important device in numerous field of signal processing, including communication, array processing, speech processing and music technology. An excellent survey of the fractional delay filter design is presented in tutorial paper [6]. Based on this useful and well-documented device, we will establish the relation between the comb filter design problem and the fractional delay filter design problem. As a result, the comprehensive design tools of the fractional delay filter in the literature can be applied to design comb filter directly.

\section{COMB FILTER DESIGN USING FRACTIONAL SAMPLE DELAY}

Generally, the input signal of comb filter has the following form:

$$
x(n)=s(n)+\sum_{k=0}^{M} A_{k} \sin \left(k \omega_{0} n+\phi_{k}\right)
$$

$$
=s(n)+I(n)
$$

where $s(n)$ is the desired signal and $I(n)$ is harmonic interference with fundamental frequency $\omega_{0}$. In order to extract $s(n)$ from the corrupted signal $x(n)$ undistortedly, the specification of ideal comb filter is given by

$$
H_{d}(\omega)=\left\{\begin{array}{ll}
0 & \omega=k \omega_{0} \\
1 & \text { otherwise }
\end{array} \quad k=0,1, \cdots, M\right.
$$

The purpose of this paper is to design a filter such that its frequency response approximates $H_{d}(\omega)$ as well as possible. To achieve this purpose, we first show that the harmonic interference $I(n)$ satisfies the following property. Define the fractional sample delay $D=\frac{2 \pi}{\omega_{0}}$ which is the period of the harmonic interference $I(n)$, then we have

$$
I(n)=I(n-D)
$$

This expression tells us that $I(n)$ is equal to its delayed version $I(n-D)$. Thus, if the signal $x(n)$ passes through the filter $H(z)=1-z^{-D}$, then its output $y(n)$ is given by

$$
\begin{aligned}
y(n) & =x(n)-x(n-D) \\
& =s(n)-s(n-D)
\end{aligned}
$$

Obviously, the harmonic interference has been eliminated in the output $y(n)$. However, $y(n)$ is not equal to $s(n)$, i.e., some distortion is included in the signal $y(n)$. In order to explain this phenomenon, Fig. 1 shows the frequency response of the filter $H(z)=1-z^{-D}$ and desired frequency response $H_{d}(\omega)$ defined in (2) with $\omega_{0}=0.22 \pi$ and $M=4$. Note that we usually choose $M=\left\lfloor\frac{\pi}{\omega_{0}}\right\rfloor$ which denotes the largest integer smaller than or equal to $\frac{\pi}{\omega_{0}}$. It is clear that both responses have the same positions of stop-band notches, but they have a large difference in the passband. In order to remove this distortion, a compensation procedure is performed as follows: It is easy to show that the zeros of the filter $H(z)=1-z^{-D}$ are given by

$$
z_{k}=e^{j \frac{2 \pi}{D} k} \quad k=\text { any integer }
$$

For all zeros $z_{k}$, we introduce the poles

$$
p_{k}=\rho e^{j \frac{2 \pi}{D} k} \quad k=\text { any integer }
$$

to eliminate the distortion in the passband of the frequency response of $H(z)=1-z^{-D}$. The radius of the pole $\rho$ must satisfy the inequality $0<\rho<1$ in order to constrain the poles to be within the unit circle. After performing this 
compensation, the new transfer function of the comb filter is given by

$$
H_{c}(z)=\frac{1-z^{-D}}{1-\rho^{D} z^{-D}}
$$

The Fig.2 shows the frequency response of $H_{c}(z)$ with parameters $\omega_{0}=0.22 \pi$ and $\rho=0.99$. It is clear that the frequency response of filter $H_{c}(z)$ approximates $H_{d}(\omega)$ very well. In fact, $H_{c}(z)$ becomes an ideal comb filter when pole radius $\rho$ approaches unity. Moreover, the direct form implementation of IIR comb filter $H_{c}(z)$ in eq( 7$)$ only requires a fractional sample delay $z^{-D}$. When $D$ is an integer, the delay $z^{-D}$ is implementable without requiring any design. However, when $D$ is not an integer, we need to design fractional sample delay $z^{-D}$. In [6], a comprehensive review of FIR and allpass filter design techniques for approximation of fractional delay has been presented. Thus, we can directly use these well-documented techniques to design $z^{-D}$. Now, two examples are provided to illustrate the performance of the method. One concerns FIR design case, the other is IIR allpass filter case.

Example 1: FIR fractional delay case

In this example, we use Lagrange interpolation method to design an FIR filter for approximating a given fractional delay $z^{-D}[6]$. In this method, the delay $z^{-D}$ is approximated by

$$
z^{-D} \approx \sum_{n=0}^{N} h(n) z^{-n}
$$

where filter coefficients $h(n)$ have the explicit form as:

$$
h(n)=\prod_{k=0, k \neq n}^{N} \frac{D-k}{n-k} \quad n=0,1, \cdots, N
$$

When the parameters are chosen as $\omega_{0}=0.22 \pi, \rho=0.99$ and $N=16$, the frequency response of $H_{c}(z)$ is shown in Fig.3. It is clear that the comb filter has an excellent approximation at low frequency because the Lagrange interpolation design is a maximally flat design at frequency $\omega=0$.

Example 2: Allpass fractional delay case

In this example, we use the maximally flat group delay allpass filter to approximate a given fractional delay $z^{-D}$ [6]. In this case, the $z^{-D}$ is approximated by

$$
z^{-D} \approx \frac{a_{N}+a_{N-1} z^{-1}+\cdots+a_{1} z^{-(N-1)}+z^{-N}}{1+a_{1} z^{-1}+\cdots+a_{N-1} z^{-(N-1)}+a_{N} z^{-N}}
$$

If the positive real number $D$ is splitted an integer $N$ plus a fractional number $d$, i.e, $D=N+d$, the filter coefficients $a_{k}$ is given by

$$
a_{k}=(-1)^{k} C_{k}^{N} \prod_{n=0}^{N} \frac{D-N+n}{D-N+k+n}
$$

where $C_{k}^{N}=\frac{N !}{k !(N-k) !}$ is a binomial coefficient. The Fig.4 shows the frequency response of the comb filter in this design if the parameters are chosen as $\omega_{0}=0.22 \pi, \rho=0.99$. It is clear that the specification is well satisfied at low frequency.

\section{COMB FILTER DESIGN BASED ON CONSTRAINED FRACTIONAL DELAY FILTER DESIGN}

Although the design methods in the example 1 and example 2 provide two excellent approximations to the ideal comb filter, the frequency responses at harmonic frequencies $k \omega_{0}$ are not exactly zero valued. This result makes the harmonic interference $I(n)$ can not be eliminated clearly by the designed comb filter. In order to remove this drawback, some suitable constraints need to be incorporated in the design of fractional sample delay $z^{-D}$. In the following, the cases of FIR filter and allpass filter will be described in details.

3.1: FIR Fractional Delay Filter Design:

In this subsection, we will design an FIR filter to approximate the fractional smaple delay $z^{-D}$. The transfer function of a causal $N$ th order FIR filter can be represented as

$$
H(z)=\sum_{n=0}^{N} h(n) z^{-n}
$$

The frequency response of the FIR filter is given by

$$
H(\omega)=\mathbf{h}^{t} \mathbf{e}(\omega)=\mathbf{e}^{t}(\omega) \mathbf{h}
$$

where vectors $\mathbf{h}$ and $\mathbf{e}(\omega)$ are

$$
\begin{aligned}
\mathbf{h} & =[h(0) h(1) \cdots h(N)]^{t} \\
\mathbf{e}(\omega) & =\left[1 e^{-j \omega} \cdots e^{-j N \omega}\right]^{t}
\end{aligned}
$$

For fractional delay filter design, the desired frequency response $F_{d}(\omega)$ is chosen as $e^{-j D \omega}$. In this paper, the filter coefficients $h$ are obtained by minimizing the following least squares error:

$$
J(\mathbf{h})=\int_{\omega \in\left(R+\cup R^{-}\right)}\left|H(\omega)-F_{d}(\omega)\right|^{2} d \omega
$$

where frequency bands $R^{+}=[0, \alpha \pi]$ and $R^{-}=[-\alpha \pi, 0]$. Using the conjugate symmetric property of $H(\omega)$ and $F_{d}(\omega)$, the error $J(\mathbf{h})$ can be rewritten as the quadratic form:

$$
J(\mathbf{h})=\mathbf{h}^{t} \mathbf{Q h}-2 \mathbf{h}^{t} \mathbf{p}+c
$$

where matrix $\mathbf{Q}$, vector $\mathbf{p}$, and scalar $c$ are real and given by

$$
\begin{aligned}
\mathbf{Q} & =2 \int_{\omega \in R^{+}} \operatorname{Re}\left(\mathbf{e}(\omega) \mathbf{e}^{H}(\omega)\right) d \omega \\
\mathbf{p} & =2 \int_{\omega \in R^{+}} \operatorname{Re}\left(F_{d}(\omega) \mathbf{e}^{*}(\omega)\right) d \omega \\
c & =2 \int_{\omega \in R^{+}}\left|F_{d}(\omega)\right|^{2} d \omega=2 \alpha \pi
\end{aligned}
$$

The $H$ denotes the Hermitian conjugate transpose operator, and $\operatorname{Re}($.$) stands for the real part of a complex number.$ In order to make comb filter be exactly zero valued at the harmonic frequencies $k \omega_{0}$, the following constraints are considered in the design procedure:

$$
H\left(k \omega_{0}\right)=e^{-j D k \omega_{0}} \quad k=0,1, \cdots, M
$$


where $M=\left\lfloor\frac{\pi}{\omega_{0}}\right\rfloor$. After some maniputation, these constraints can be written in vector matrix form $\mathbf{C h}=\mathbf{f}$, where real valued matrix $\mathbf{C}$ and vector $\mathbf{f}$ are given by

$$
\begin{aligned}
\mathbf{C} & =\left[\mathbf{e}(0), \operatorname{Re}\left(\mathbf{e}\left(\omega_{0}\right)\right), \operatorname{Im}\left(\mathbf{e}\left(\omega_{0}\right)\right), \cdots, \operatorname{Im}\left(\mathbf{e}\left(M \omega_{0}\right)\right)\right]^{t} \\
\mathbf{f} & =\left[1, \cos \left(D \omega_{0}\right),-\sin \left(D \omega_{0}\right), \cdots,-\sin \left(D M \omega_{0}\right)\right]^{t}
\end{aligned}
$$

where $\operatorname{Im}($.$) stands for the imaginary part of a complex$ number. Using tne Lagrange multiplier method, the optimal solution of this constrained problem is given by

$$
\mathbf{h}=\mathbf{Q}^{-1} \mathbf{p}-\mathbf{Q}^{-1} \mathbf{C}^{t}\left(\mathbf{C Q} \mathbf{Q}^{-1} \mathbf{C}^{t}\right)^{-1}\left[\mathbf{C Q} \mathbf{Q}^{-1} \mathbf{p}-\mathbf{f}\right]
$$

Now, we use an example to examine the performance of this design method.

\section{Example 3: Constrained FIR Filter Case}

In this example, the design parameters are chosen as $\alpha=$ $0.9, \omega_{0}=0.22 \pi, \rho=0.999$ and $N=16$. The frequency response of the designed comb filter $H_{c}(z)$ is shown in Fig. 5. It is clear that the frequency response of the comb filter is exactly zero valued at harmonic frequencies $k \omega_{0}$ and almost has unity gain at the remaining frequencies.

\section{2: Allpass Fractional Delay Filter Design:}

It is easy to show that the phase response $\theta_{A}(\omega)$ of the all.pas filter in eq $(10)$ can be written as

$$
\theta_{A}(\omega)=-N \omega+2 \arctan \left(\frac{\sum_{k=1}^{N} a_{k} \sin (k \omega)}{1+\sum_{k=1}^{N} a_{k} \cos (k \omega)}\right)
$$

The purpose of this subsection is to design an allpass filter such that the $\theta_{A}(\omega)$ approximates the prescribed phase response $-D \omega$, that is, we want to achieve the following specification:

$$
\theta_{A}(\omega)=-D \omega \quad \omega \in[0, \alpha \pi]
$$

Substitute eq(19) into eq(20), we obtain the expression [7]:

$$
\mathbf{a}^{t} \mathbf{b}(\omega)=-\sin (\beta(w))
$$

where $\beta(\omega)=\frac{-1}{2}(-D \omega+N \omega)$, and two vectors

$$
\begin{aligned}
\mathbf{a} & =\left[\begin{array}{ll}
a_{1} & a_{2} \cdots a_{N}
\end{array}\right]^{t} \\
\mathbf{b}(\omega) & =[\sin (\beta(\omega)+\omega) \cdots \sin (\beta(\omega)+N \omega)]^{t}
\end{aligned}
$$

In this paper, we will minimize the following least squares error to obtain optimal filter coefficients a:

$$
\begin{aligned}
J(\mathbf{a}) & =\int_{0}^{\alpha \pi}\left|\mathbf{a}^{t} \mathbf{b}(\omega)+\sin (\beta(\omega))\right|^{2} d \omega \\
& =\mathbf{a}^{t} \mathbf{Q} \mathbf{a}-2 \mathbf{p}^{t} \mathbf{a}+c
\end{aligned}
$$

where matrix $\mathbf{Q}$, vector $\mathbf{p}$ and scalar $c$ are given by

$$
\begin{aligned}
\mathbf{Q} & =\int_{0}^{\alpha \pi} \mathbf{b}(\omega) \mathbf{b}(\omega)^{t} d \omega \\
\mathbf{P} & =-\int_{0}^{\alpha \pi} \mathbf{b}(\omega) \sin (\beta(\omega)) d \omega \\
c & =\int_{0}^{\alpha \pi} \sin (\beta(\omega))^{2} d \omega
\end{aligned}
$$

In order to make comb filter be exactly zero valued at harmonic frequencies $k \omega_{0}$, the following constraints are incorporated in the design:

$$
\mathbf{a}^{t} \mathbf{b}\left(k \omega_{0}\right)=-\sin \left(\beta\left(k w_{0}\right)\right) \quad k=1,2, \cdots, M
$$

where $M=\left\lfloor\frac{\pi}{\omega_{0}}\right\rfloor$. After some maniputation, these constraints can be written in vector matrix form $\mathrm{Ca}=\mathrm{f}$, where real valued matrix $\mathbf{C}$ and vector $\mathbf{f}$ are given by

$$
\begin{aligned}
\mathbf{C} & =\left[\mathbf{b}\left(\omega_{0}\right), \mathbf{b}\left(2 \omega_{0}\right), \cdots, \mathbf{b}\left(N \omega_{0}\right)\right]^{t} \\
\mathbf{f} & =\left[-\sin \left(\beta\left(\omega_{0}\right)\right),-\sin \left(\beta\left(2 \omega_{0}\right)\right), \cdots,-\sin \left(\beta\left(N \omega_{0}\right)\right)\right]^{t}
\end{aligned}
$$

Using tne Lagrange multiplier method, the optimal solution of this constrained problem can be obtained as FIR design case. Finally, we use an example to investigate the performance of this design method.

Example 4: Constrained Allpass Filter Case

In this example, the design parameters are chosen as $\alpha=0.9, \omega_{0}=0.22 \pi, \rho=0.999$ and $N=\left\lfloor\frac{2 \pi}{\omega_{0}}\right\rfloor=9$, The frequency response of $H_{c}(z)$ is shown in Fig.6. It is clear that the frequency response of the designed comb filter is exactly zero valued at harmonic frequencies $k \omega_{0}$ and almost has unity gain at the remaining frequencies.

\section{CONCLUSION}

In this paper, a new comb filter design method using fractional sample delay has been presented. First, the specification of the comb filter design is transformed into that of fractional delay filter design. Then, the FIR and allpass filter design techniques are directly used to design fractional delay filter with transformed specification. Next, we develop a constrained fractional delay filter design approach to improve the performance of the direct design method. Finally, several design examples are demonstrated to illustrate the effectiveness of this new design approach.

\section{REFERENCES}

[1] J.A. VAN ALSTE and T.S. Schilder, "Removal of Based-Line Wander and Power-Line Interference from the ECG by an Efficient FIR. Filter with Reduced Number of Taps", IEEE Trans. Biomedical Engineering, Vol.BME-32, pp.1052-1060, Dec. 1985.

[2] J.D. Wang and H.J. Trussell, "Adaptive Harmonic Noise Cancellation with an Application to Distribution Power Line Communication", IEEE Trans. Communications, Vol.36, pp.875-884, July 1988.

[3] A. Nehorai and B. Porat, "Adaptive Comb Filtering for Harmonic Signal Enhancement", IEEE Trans. Acoust. Speech and Signal Processing, Vol.ASSP-24, pp.11241138, Nov. 1986.

[4] Y.K. Jang anf J.F. Chicharo, "Adaptive IIR Comb Filter for Harmonic Signal Cancellation", Int. J. Electronics, Vol.75, pp.241-250, 1993.

[5] S.C. Pei and C.C. Tseng, "Elimination of AC Interference in Electrocardiogram Using IIR Notch Filter with Transdient Suppression", IEEE Trans. Biomedical Engineering, Vol.42, pp.1128-1132, Nov. 1995.

[6] T.I. Laakso, V. Valimaki, M. Karjalainen and U.K. Laine, "Splitting the Unit Delay: Tools for Fractional Delay Filter Design", IEEE Signal Processing Magazine", pp.30-60, Jan. 1996.

[7] M. Lang and. T.I. Laakso, "Simple and Robust Method for the Design of Allpass Filters Using Least Squares Phase Error Criterion", IEEE Trans. Circuits Syst. II: Analog and Digital Signal Processing, Vol.41, pp.40-48, Jan. 1994. 


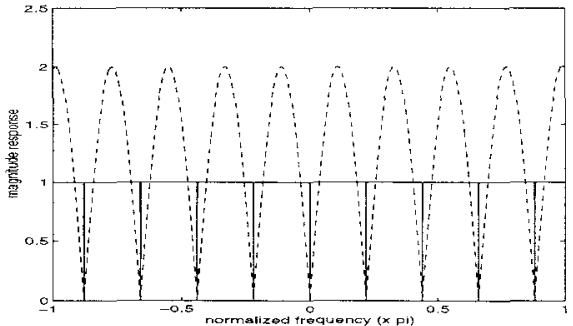

Figure 1: The frequency responses of the filter $H(z)=1-z^{-D}$ (dashed line) and the ideal comb filter (solid line).

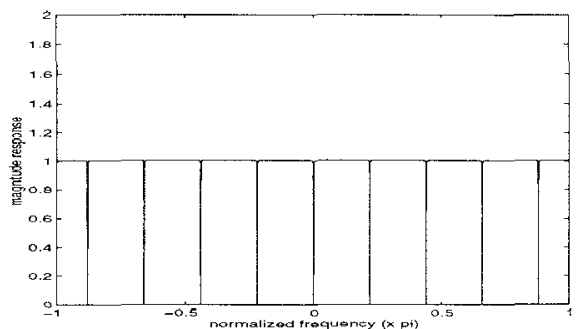

Figure 2: The magnitude response of the comb filter $H_{c}(z)$.

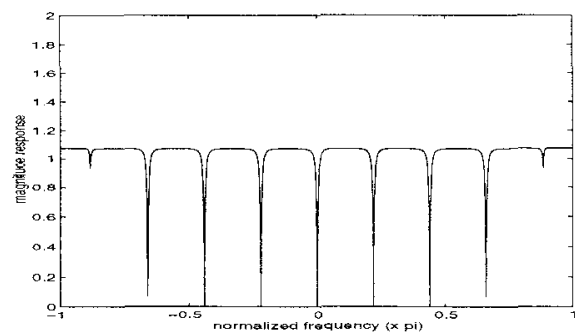

Figure 3: The magnitude response of the designed comb filter in example 1.

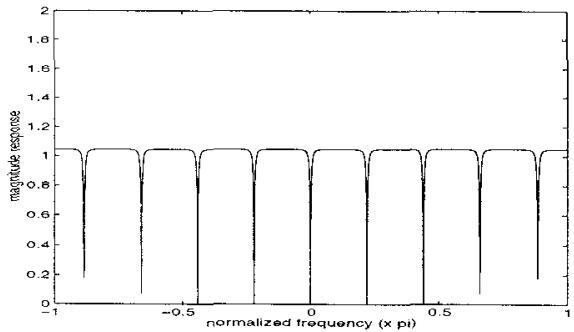

Figure 4: The magnitude response of the designed comb filter in example 2.

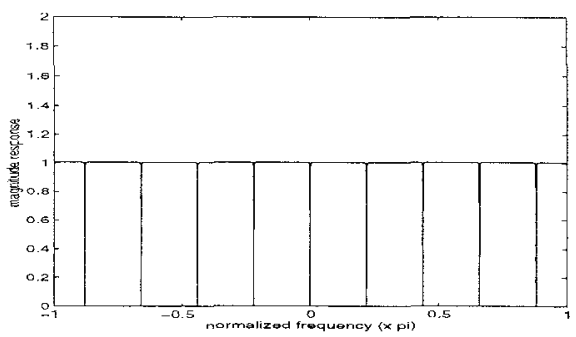

Figure 5: The magnitude response of the designed comb filter in example 3 .

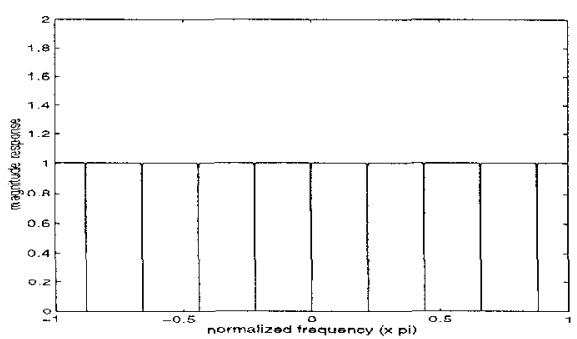

Figure 6: The magnitude response of the designed comb filter in example 4. 